\title{
DISTRIBUCION SUPERFICIAL DE ALGUNOS PARAMETROS HIDROLOGICOS FISICOS Y QUIMICOS EN EL ESTERO DE PUNTA BANDA, B. C. EN PRIMAVERA Y VERANO.
}

\author{
Por:

\section{Raúl Celis Ceseña y Saúl Alvarez Borrego*} \\ Ciencias Marinas Vol. 2 Nulm.I \\ * Ocupación Actual: Director del Centro de Investigación Científica y Educación Supe- \\ rior de Ensenada, B. C.
}

\section{RESUMEN}

\begin{abstract}
Durante primavera y verano de 1973 se realizaron cinco muestreos de superficie en el Estero de Punta Banda. En el presente trabajo se presentan y discuten los resultados sobre salinidad, temperatura, concentración de oxigeno disuelto, por ciento de saturación de oxígeno disuelto y $\mathrm{pH}$. Esto complementa el estudio de la variación a través de un cicla anual de las condiciones hidrológicas del Estero, iniciado por Acosta Ruiz y Alvarez Borrego (1974). El principal objetivo ha sido el de conocer las condiciones ecológicas para poder decidir sobre especies a cultivarse en el Estero. El Estero es un antiestuario, con salinidad y temperatura mós elevadas hacia el interior que en la boca durante todo el año. La salinidad máxima, registrada en julio, fue $37.36 \%$ y y la temperatura máxima, registrada también en julio, fue $26.0^{\circ} \mathrm{C}$. Con relación al desarrollo de ostricultivos se euncluye que los rangos de variación de salinidad, temperatura, concentración de oxígeno disuelto y $\mathrm{pH}$ no son limitantes para el crecimiento de Crassostrea gigas, Ostrea lurida y Ostrea edulis, pero la salinidad no permite el cultivo de Crassostrea virginica. La temperatura del extremo interno en verano es adecuada para el desove natural de Grassostrea gigas y Ostrea edulis.
\end{abstract}

\section{ABSTRACT}

Surface samples were taken in five trips to the Estero de Punta Banda, during Spring and Summer of 1973. In the present manuscript results of temperature, salinity, dissolved oxygen concentration, percent saturation of dissulved oxygen and $\mathrm{pH}$ are presented and discussed. This study is the continuation of the num started by Acosta Ruiz and Alvarez Borrego (1974) on the ann....' cycle variation of the hydrological conditions of the Etero. The main objective is to know the ecological condi. tions in order to decide on which species to cultivate in the Estere. The Esters is ath antiestuarine system, with salinity and temperature higher in the ifterior than at the mouth during all year. The maximum salinity and Femperature were $37.36 \%$ and $26.0{ }^{\circ} \mathrm{C}$, respectively, both registered in July. With relation to the development of oyster culture, we conslude that the salinity, temperature, concentration of dissolved oxy. gen and $\mathrm{pH}$ ranges are not limitant for the growth of Crassostrea gigas, Ostred lutida and Ostrea edulis; but salinity does not allow the eulture of Crassustrea virginica. At the internal end, temperature is adequate for natural spawning of Crassostrea gigas and Ostrea edulis, during summer.

\section{INTRODUCCION}

Con el objetivo de conocer la ecología del Estero de Punta Banda para decidir sobre las especies a cultivarse en su interior, Acosta Ruiz y Alvarez Borrego (1974) estudiaron las condiciones hi- drológicas en el período de octubre de 1972 a marzo de 1973. Con el fin de completar el estudio del ciclo anual de variación de dichas condiciones hidroló. gicas presentamos en este trabajo los re. sultados obtenidos de mayo a agosto de 1973. 
El Estero de Punta Banda es una laguna costera localizada a lo largo de la orilla sureste de la Bahía de Todos Santos, B. C. Está caracterizado por un canal en forma de " $L$ "; $y$ está separado de la Bahía por una barra arenosa que se extiende de Punta Banda hacia el inoreste, con un poco más de siete kilómetros de longitud (Fig. 1). Ya que los antecedentes se describen con detalle por Acosta Ruiz y Alvarez Borrego (1974), no consideramos necesario repetirlos aquí. Además Lara Lara y Alvarez Borrego (1975, este númerol, presentan algunas consideraciones sobre las lagunas costeras con referencia a su potencialidad para el desarrollo de maricultivos.

La influencia de las condiciones hidrológicas del Estero en la Bahía de Todos Santos es importante para la ecología de esta última. Los isotermas horizontales para diferentes niveles en la Bahía, señalan una influencia, variable en extensión e intensidad, del Estero de Punta Banda, acentuándose en verano (Cabrera Muro, 1974). Contreras Rivas (1973) concluyó que el área de influencia del Estero sobre la Bahía abarca la zona comprendida en un perímefro de hasta $6 \mathrm{~km}$ de la boca del Estero hacia mar adentro.

La boca del estero varía en amplitud de acuerdo al aumento o disminución de la cantidad de sedimentos depositados por el oleaje. En verano la anchura de la boca es de menos de 200 metros, siendo mayor en invierno.

Acosta Ruiz y Alvarez Borrego (1974) registraron los siguientes rangos para las 'variables hidrológicas:

\section{OTONO}

$\begin{array}{lcccc} & \mathrm{T}^{\circ} \mathrm{C} & \text { Salinidad }(\% / \mathrm{oo}) & \text { Oxigeno }(\mathrm{ml} / \mathrm{I}) & \mathbf{p H} \\ \text { Máximo } & 21.2 & 35.30 & 5.77 & 8.27 \\ \text { Mínimo } & 16.0 & 33.53 & 3.92 & 8.00 \\ & & & & \\ \text { INVIERNO } & & & & \\ & & & & \\ & \mathrm{T}^{\circ} \mathrm{C} & \text { Salinidad }(\% / \mathrm{oo}) & \text { Oxígeno }(\mathrm{ml} / \mathrm{ll} & \mathbf{p H} \\ \text { Máximo } & 16.2 & 34.21 & 7.04 & 8.50 \\ \text { Minimo } & 12.0 & 33.25 & 3.39 & 8.00\end{array}$

\section{METODOS DE OBTENCION DE DATOS}

Se realizaron cinco muestreos de superficie, dos en primavera $(4$ y 31 de mayol, y tres en verano $(1$ y 30 de julio, y 30 de agosto). Las estaciones de muestreo se localizaban por estima, apoyándose en puntos fijos en la costa. El error estimado de la localización se considera no mayor de 10 metros. La duración promedio de muestreo fue de 4 horas. En cada muestreo se determinaba la temperatura, $\mathrm{pH}$ y las condiciones meteorológicas, y se colectaban muestras de agua para su posterior análisis en el laboratorio para determinar la salinidad y la concentración de oxígeno disuelto. Las muestras de oxígeno se fijaban inmediatamente después de colectarlas. Los métodos de análisis fueron los mismos utilizados por Acosta Ruiz y Alvarez Borrego (1974).

Debido a que los datos tienen un comportamiento similar en general, se

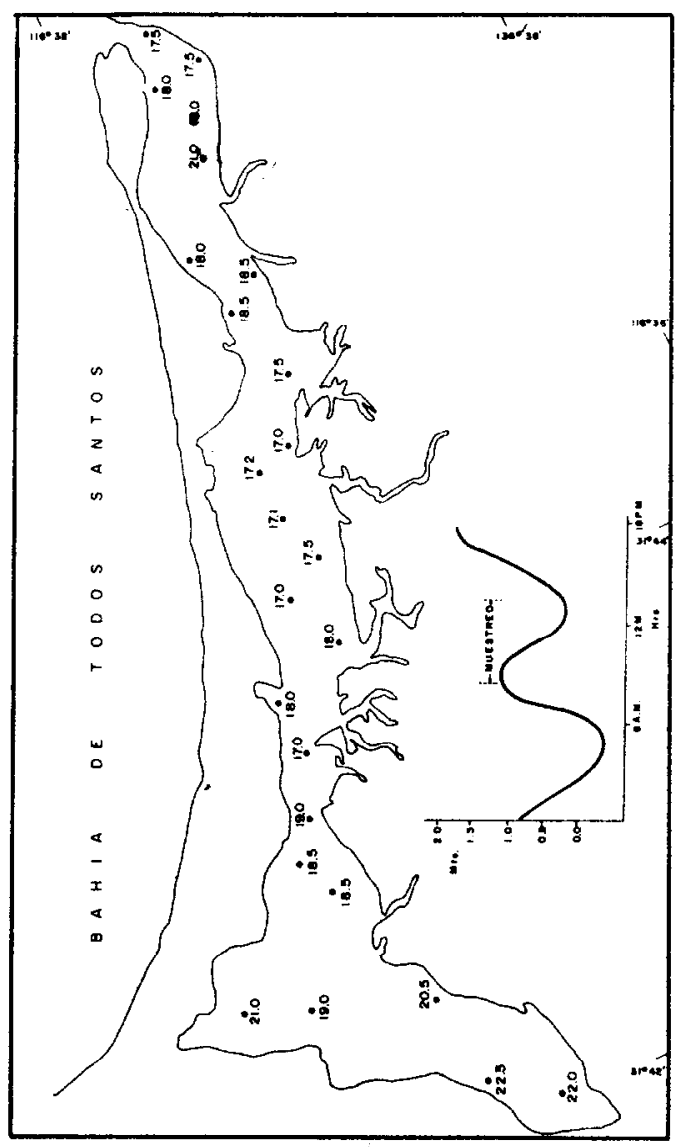

Fig. 1. Distribución superficial de To C para mayo. La curva de marea se tomó del calendorio gráfico de lo Secretaria de Marina. 
tomaron dos muestreos representativos, uno para primavera $y$ otro para verano (4. de mayo y 30 de julio), para presentarlos y discutirlos en el presente escrito.

\section{RESULTADOS}

\section{Muestreo I (4 de mayo):}

Hacia el interior del Estero los valores de temperatura pasan por un mínimo en la parte central, incrementóndose luego en dirección a la cabeza. El valor mínimo registrado fue de $17.0^{\circ} \mathrm{C}$; y el máximo fue de $22.5^{\circ} \mathrm{C}$ cerca de la cabeza del Estero (Fig. 1).

Los valores de salinidad se mantienen homogéneos de la boca a la mitad del estero aproximadamente; de ahí en adelante aumentan en dirección a la cabe$z a ;$ el mínimo fue de $33.86 \%$ en la parte media del Estero y el máximo fue de $35.48 \%$ registrado en la cabeza (Fig. 2).

Los valores de concentración de oxigeno disuelto estón distribuidos irregularmente (Fig. 3), aunque en general presentan características opuestas a la temperatura y salinidad; es decir los valores en general disminuyen en dirección a la cabeza del Estero. El valor mínimo fue de $5.32 \mathrm{ml} / \mathrm{l}$ registrándose en la cabeza, y el valor máximo fue de 6.65 $\mathrm{ml} / \mathrm{l}$ localizado cerca de la boca.

La distribución del porciento de saturación de oxígeno es muy similar a la concentración de oxígeno disuelto (Figs. 3 y 4). La distribución de estos va; lores es irregular. Podemos decir que los más altos están cerca a la boca y los más bajos cerca a la cabeza del Este-

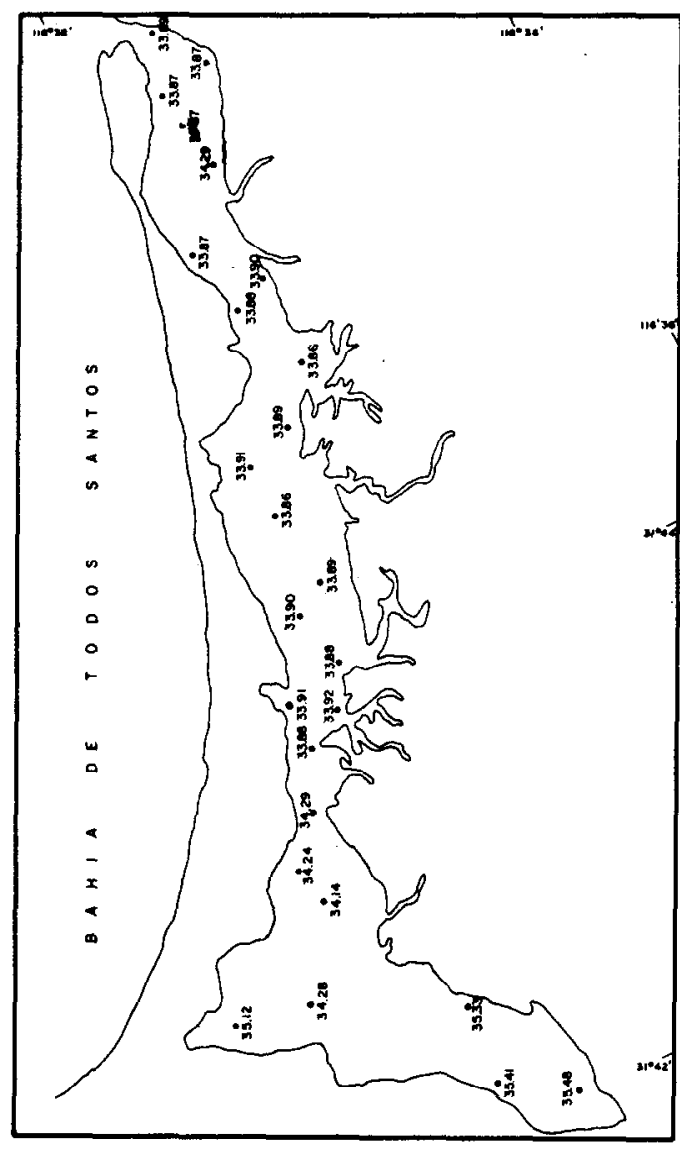

Fig. 2. Distribución superficial de solinidad $\left(S^{\prime \prime} / \infty 0\right)$ para mayo.

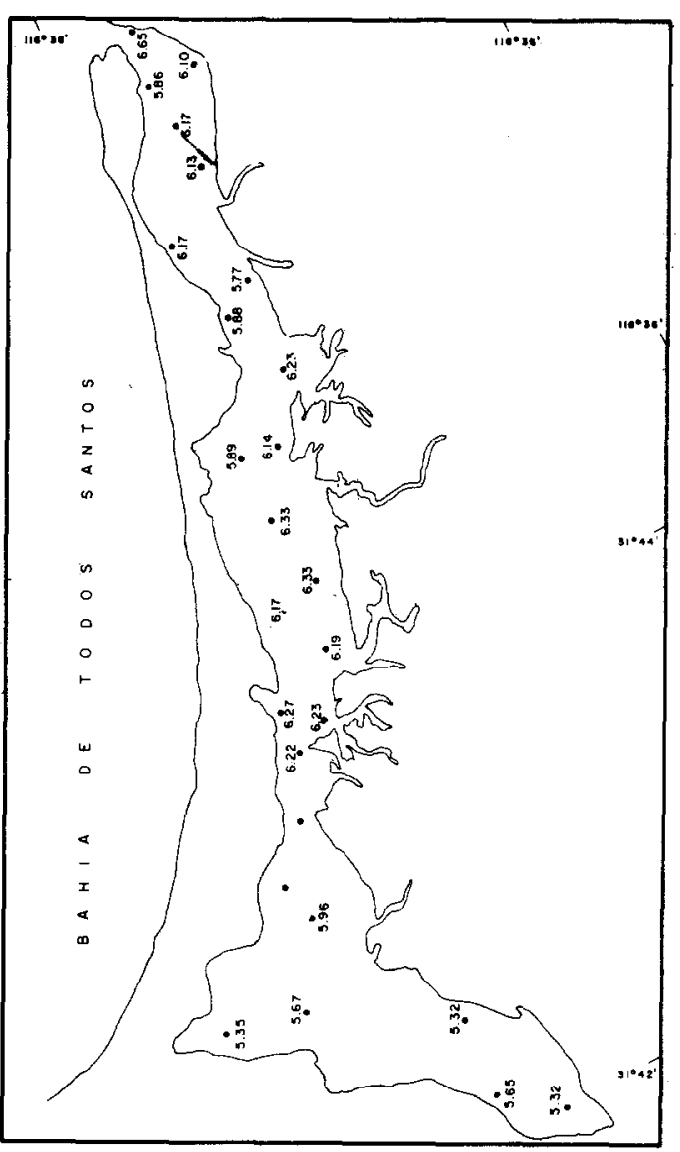

Fig. 3. Distribución superficial de la concentración de oxígeno disuelfo $(\mathrm{m} / / \mathrm{ll})$ paro mayo. 
ro. En la parte media del Estero encontramos valores altos laproximadamente $115 \%$. El máximo se encontró cerca de la boca con un valor de $121 \%$; y el minimo cerca de la cabeza, siendo de $103 \%$

El pH superficial presenta características similares a la distribución de la concentración de oxígeno disuelto, los valores disminuyen en dirección a la cabeza del Estero. Cerca a la boca hay un gradiente transversal con los valores disminuyendo hacia la barra; en la parte central del Estero los valores son homogéneos. El valor minimo registrado, fue de 7.90 y corresponde con el mínimo de oxígeno localizado en la cabeza; y el máximo fue de 8.30 y corresponde con el máximo de oxígeno, registrado cerca de la boca del Estero (Figs. 3 y 5 ).

\section{Muestreo II (30 de julio):}

Los valores de temperatura presentan la misma tendencia de aumentar en dirección a la cabeza del Estero (Fig. 6). De la parte media del Estero hacia la boca los valores se encuentra entre los $22.0^{\circ} \mathrm{C}$ y los $22.5^{\circ} \mathrm{C}$; pero hacia la cabeza la temperatura se incrementa alcanzando el móximo valor cerca del extremo interno, siendo de $26.0^{\circ} \mathrm{C}$; el mínimo fue de $21.0^{\circ} \mathrm{C}$ localizado cerca de la boca. En general, se registró un aumento de la temperatura superficial en todo el Estero, de mayo a julio, de aproximadamente $4^{\circ} \mathrm{C}$ (Figs. 1 y 6 ).

Los valores de salinidad aumentan en el mismo sentido de la temperatura en general hacia la cabeza del Estero (Figs. 6 y 7). De la parte media del Estero hacia la boca los valores fueron aproximadamente $33.80^{\circ} \% 0$, a excepción de un valor alto cerca de la boca. Podemos notar que en la base de la " $L$ " todos los valores estón arriba de $35.00^{\circ} / 00$. El valor mínimo registrado fue de $33.80^{\circ} / 00$. se encontró en la parte media del Estero; el máximo fue de $37.36 \%$ oo localizado en la cabeza. A diferencia de la temperatura, el aumento de la salinidad superficial, en julio con respecto a mayo, fue significativa solamente en la cabeza del Estero (Figs. 2 y 7 ).

Los valores de concentración de oxigeno disuelto están distribuidos en una forma un tanto irregular, aunque en ge-

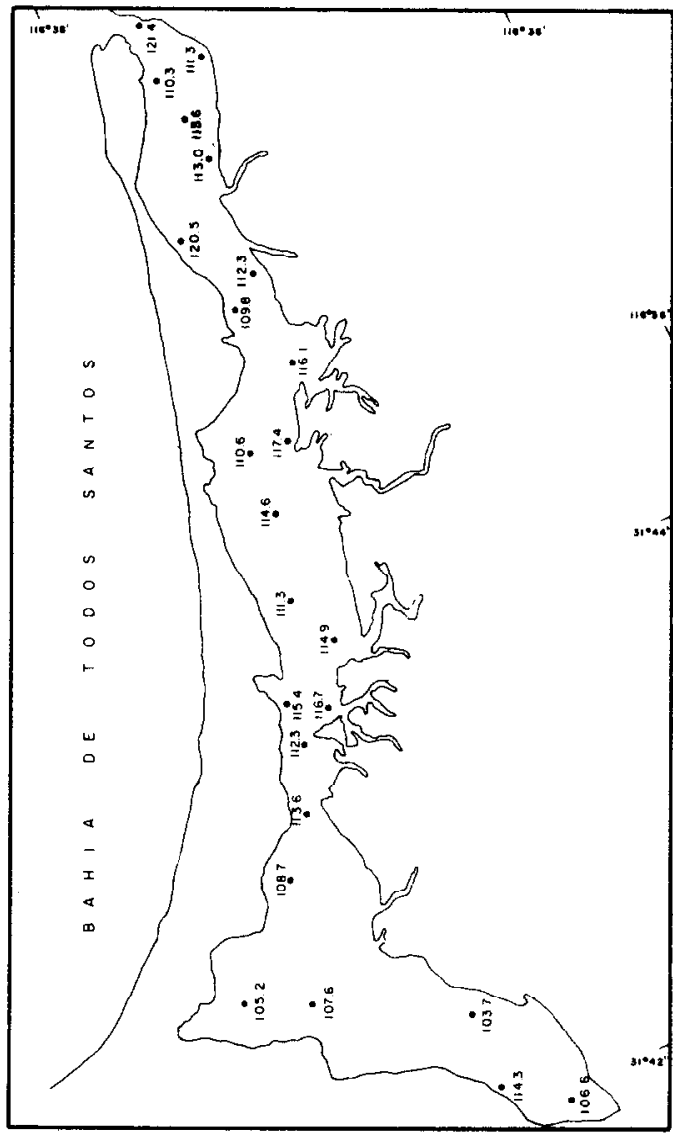

Fig. 4. Distribución superficial del $\%$ de saturación de oxígeno disuelto para mayo.

neral existen valores menores en la cabeza que en el resto del Estero (Fig. 8) el mínimo valor registrado fue de 4.15 $\mathrm{ml} / \mathrm{I}$ en el extremo interno; el valor máximo fue de $5.68 \mathrm{ml} / \mathrm{l}$ registrado en la parte media. La distribución de la concentración superficial de oxígeno disuelto disminuyó en julio, aproximadamente I $\mathrm{ml} / \mathrm{l}$, con respecto a mayo (Figs. 3 y 8).

La distribución de los valores del porciento de saturación de oxígeno es irregular (Fig. 9). Los valores bajos están en la cabeza del Estero; los valores altos se encuentran en la parte media y en la boca; el mínimo fue de $90 \%$ registrado en la cabeza; el máximo fue de $116 \%$ registrado en la boca.

El $\mathrm{pH}$ disminuye en dirección a la cabeza del Estero (Fig. 10). En la zona cercana a la boca todos los valores es- 


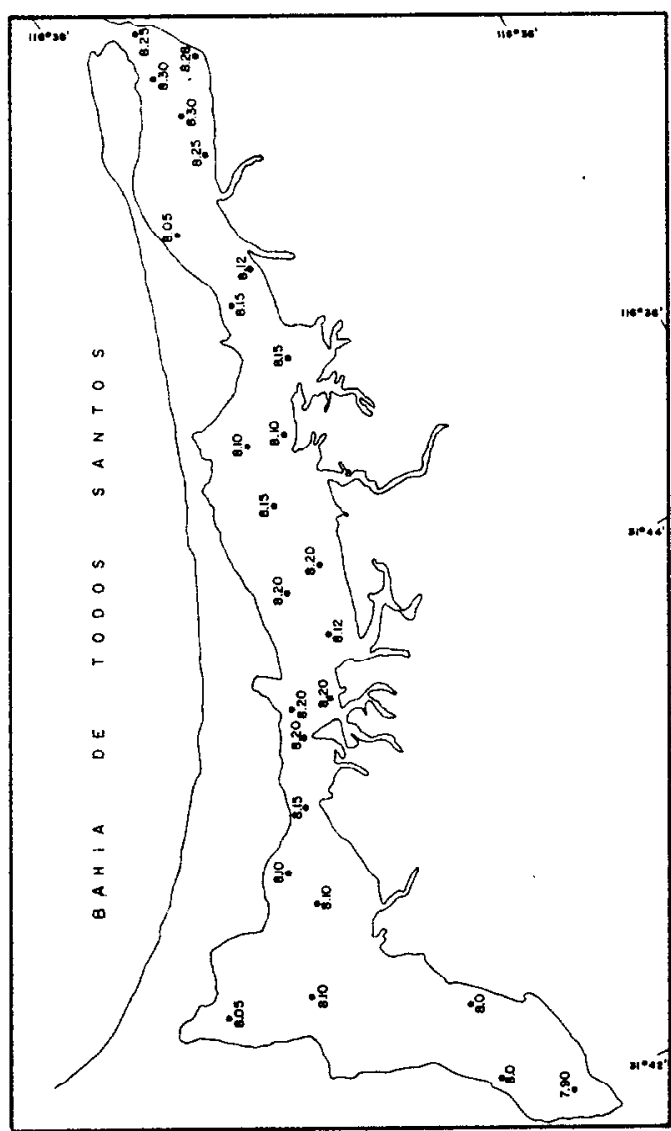

Fig 5. Distribución superficial de $\mathrm{pH}$ para moyo

tán arriba de 8.0; de la parte media al extremo interno fueron menores a 8.0. El mínimo fue de 7.77 en el extremo interno; el máximo registrado fue 8.11 cerca a la boca del Estero.

\section{DISCUSIONES}

Alvarez Borrego y Chee Barragán (no publicadol expresaron que Bahía San Quintín, que se puede considerar como una laguna costera, es un ecosistema que cuenta con una población abundante de "pastos" marinos (Zostera sp.) que le permite atrapar gran parte del material orgúnico que proviene del exterior con el flujo de las mareas. Estos autores obtuvieron esta conclusión en base a la distribución de nutrientes ffosfatos, nitratos

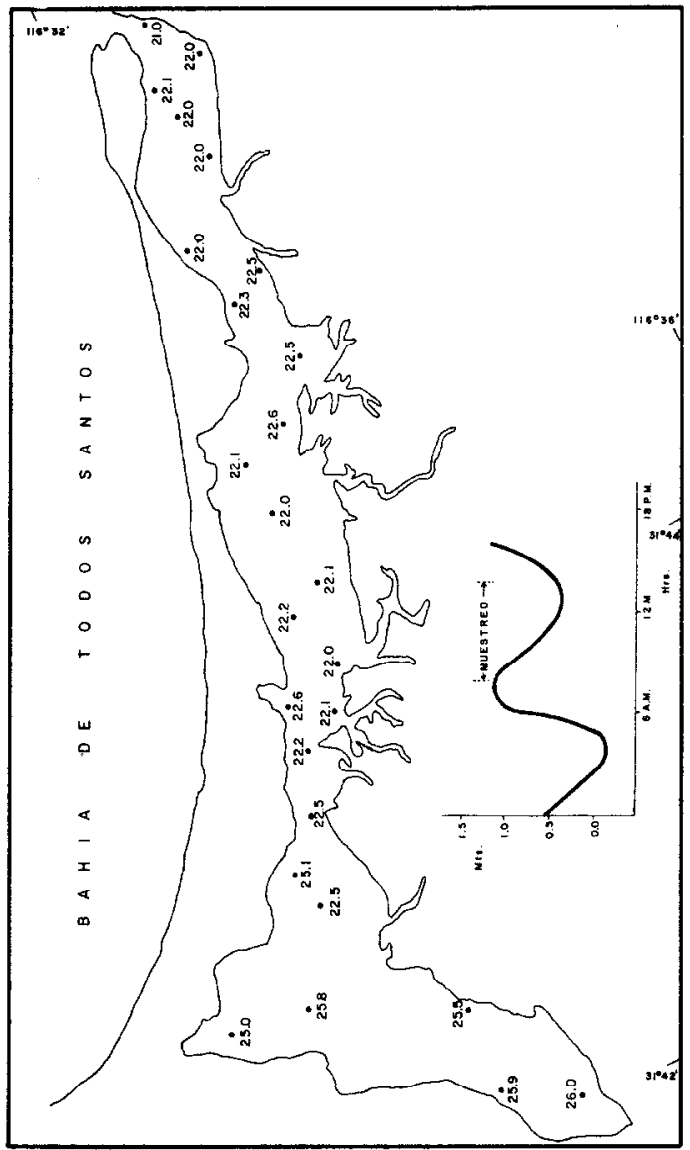

1Fig. 6. Distribución superficial de T'C para julto. La curva de marea se tomó del calendaric gráfico de la Secretaría de Marina.

y silicatos) en el interior de la bahía. El Estero de Punta Banda cuenta también con una pablación abundante de "pastos" marinos. De acuerdo con Sánchez Hernández (comunicación personal) la distribución de nutrientes en el Estero es similar a la de Bahia San Quintín, con las concentraciones aumentando de la boca hacia el extremo interno. Esto indica que el Estero de Punta Banda es también un ecosistema cuya riqueza orgánica proviene en gran parte del exterior mediante el flujo de mareas. En este tipo de ecosistemas los "pastos" marinos actúan como atenuantes de las corrientes de mareas permitiendo que el material en suspensión, orgánico e inorgánico, se deposite en el fondo, causando una concentración elevada de material orgánico principalmente en los se- 
dimentos de los extremos internos. Esto se nota también en el hecho de que la turbidez del agua aumenta hacia el extremo interno; y además la concentración de oxígeno disuelto y el $\mathrm{pH}$ disminuyen IFigs. 3, 5, 8 y 10). Estos sedimentos con alta concentración de material orgánico tienen a menudo una producción significativa de ácido sulfhídrico, que se puede apreciar por el olor de los mismos.

Las poblaciones de "pastos" marinos constituyen además un mecanismo mediante el cual este tipo de lagunas costeras va desapareciendo por azolvamiento.

Jaime Silva (1974) reportó el crecimiento del ostión japonés (Crassostrea gigas) hasta una talla comercial (aproximadamente $8 \mathrm{~cm}$ ) en solamente cuatro meses en el Estero de Punta Banda. Esta

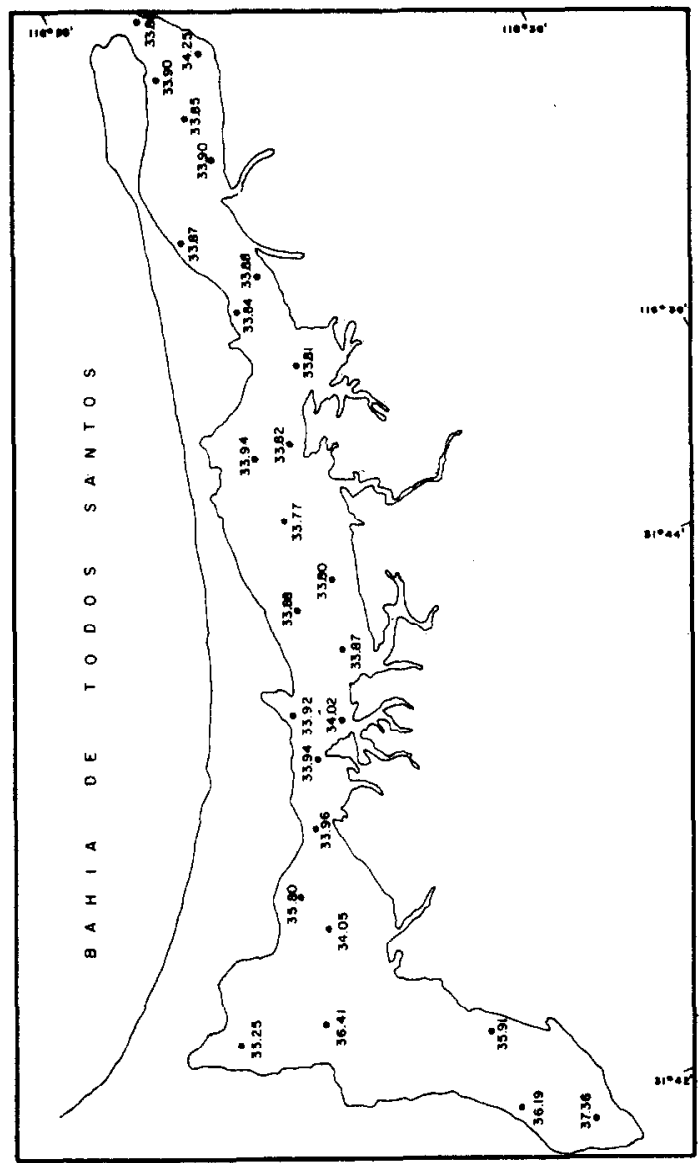

Fig. 7. Distribución superficiol de salinidad $(5 \% / 00)$ para julio. especie no nativa fue introducida en cultivo experimental en el vértice de la " $L$ " del Estero. Las conclusiones de Jaime Silva (1974) demuestran que el Estero es un lugar adecuado para cultivo de ostión japonés hasta el punto de cerrar el ciclo reproductivo con desoves naturales.

El ostión japonés requiere temperaturas mayores de $19^{\circ} \mathrm{C}$ para desovar (Bardach, Ryther, Mclarney, 1972). De acuerdo con los resultados obtenidos en este trabajo en mayo se obtuvieron temperaturas mayores de $19^{\circ} \mathrm{C}$ del vértice de la "L" hacia el extremo interno del Estero (Fig. 1), $y$ en julio se obtuvieron temperaturas mayores de $19^{\circ} \mathrm{C}$ en todo el Estero. Por lo anterior se podría utilizar el Estero como un lugar de colecta de "semilla" natural de ostión japonés, trasladando luego parte de la misma a otras aguas

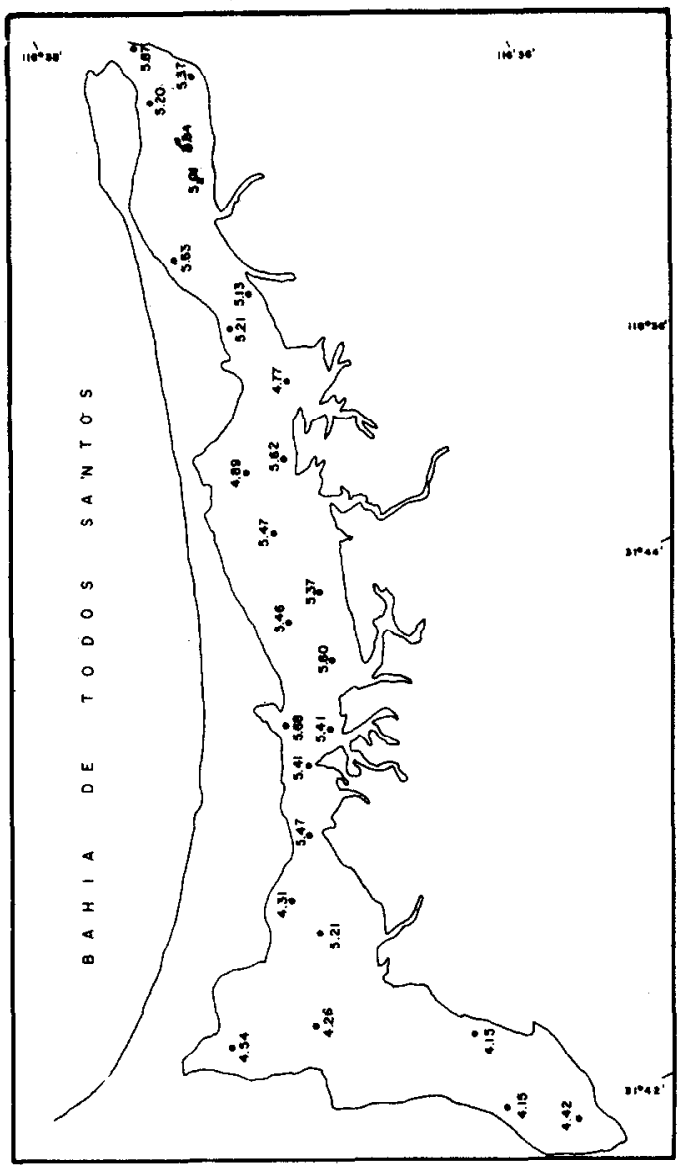

Fig. 8. Distribución superficial de la concentración de oxígeno disuelto $[\mathrm{ml} / \mathrm{l}\}$ pora julio. 


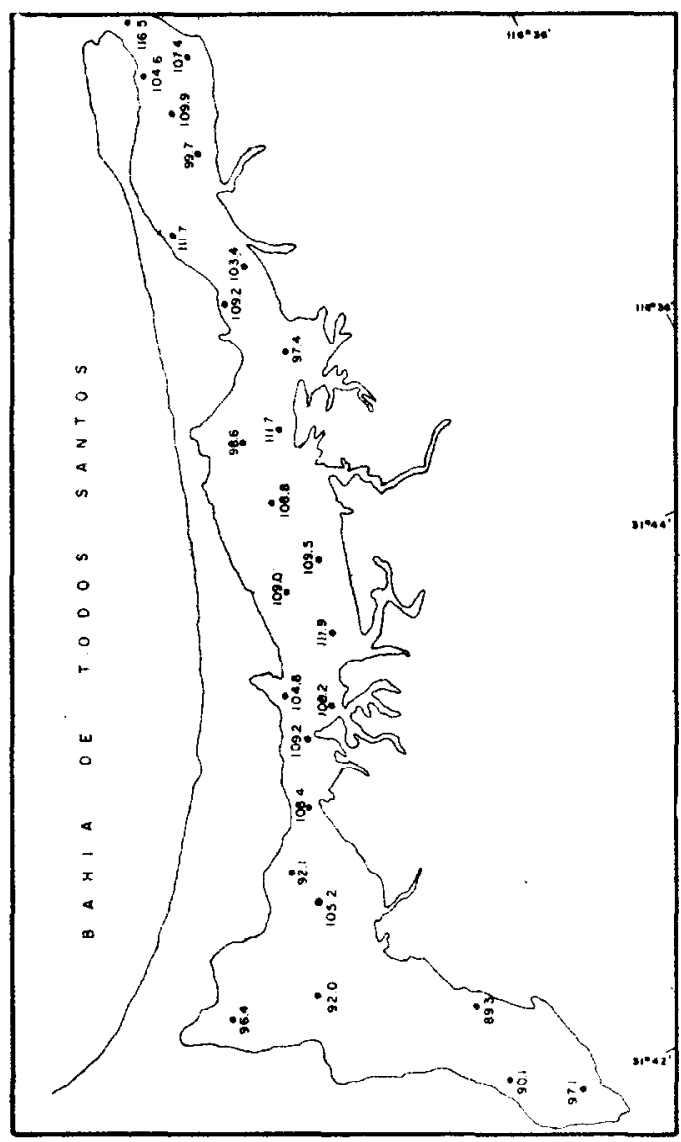

Fig. 9. Dispribución superficial del $\%$ de sọturación de oxígeno disueltó para julio.

de menor temperatura que sean adecuadas para la "engorda", como Bahía San Quintín donde no se ha cirtenido un desove natural comercialmente significativo en el proyecto de ostricultivo que actualmente está llevando a cabo la Unidad de Ciencias Marinas de la UABC Ilslas Olivares, comunicación personal).

La variación de salinidad, temperatura, oxigeno disuelto y $\mathrm{pH}$, a través de todo el año (Acosta Ruiz y Alvarez Borrego, 1974, y este trabajol es tal que no constituye limitante alguno para el desarrollo del cultivo de ostión japonés. Sin embargo, la salinidad es muy elevada para el ostión americano (Crassostrea virginica). Loosanoff (1965) menciona que esta última especie no puede desarrollarse en agua con salinidad mayor a $30^{\circ} / 00$. De acuerdo con los resultados de Acosta

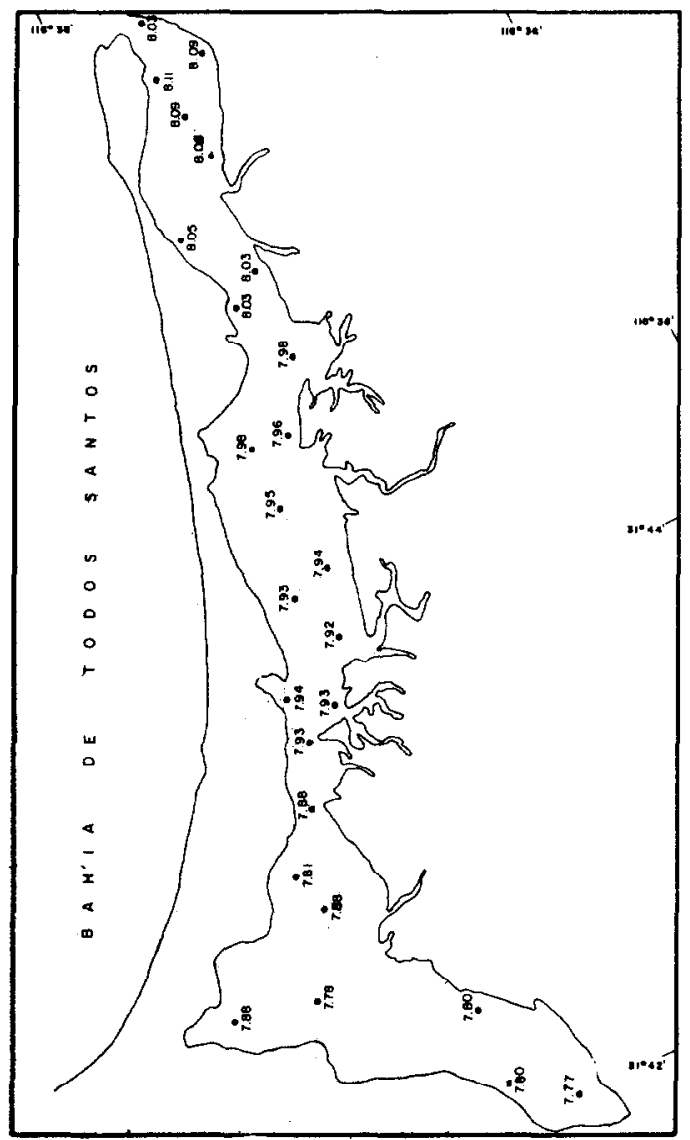

Fig. 10. Distribución superficial de $\mathrm{pH}$ para julio.

Ruiz y Alvarez Borrego (1974) y los del presente trabajo, la salinidad en el Estero es persistentemente mayor a $30 \%$, con valores máximos de alrededor de $37 \%$ en julio (Fig. 7). Ostrea lurida $y$ Ostrea edulis deben también desde el punto de vista ecológico, ser adecuadas para cultivo en el Estero, ya que soportan altas salinidades (Bardach, Ryther y McLarney, 1972).

El Estero se comporta durante todo el año como un antiestuario, con salinidad siempre mayor en el extremo interno que en la boca; es una cuenca de evaporación. Además, la temperatura es siempre mayor en el extremo interno que en la boca (Figs. 1 y 61 , aun en enero (Acosta Ruiz y Alvarez Borrego, 1974).

La distribución superficial de la concentración de oxígeno disuelto está go- 
bernada conjuntamente por procesos biológicos, intercambio gaseoso aire-agua, y el flujo y reflujo de mareas. En la zona cercana a la boca el porciento de saturación de oxígeno es mayor de 100 (Figs. 4 y 9) debido a la turbulencia provocada por el oleaje frente a la misma. En el extremo interno el porciento de saturación fue menor de 100 en julio (Fig. 9) debido a un intenso proceso de oxidación bioquímica por el alto contenido de material orgánico en suspensión, aunada a un intercambio gaseoso lento con la atmósfera. Las menores concentraciones de oxígeno registradas en julio con respecto a mayo (Figs. 3 y 8 ) se deben principalmente a la mayor temperatura de julio (Figs. 1 y b) que causaron una menor solubilidad del oxígeno y una mayor actividad de oxidación bioquímica. El $\mathrm{pH}$ proporciona en general el mismo tipo de información que la concentración de oxígeno disuelto corroborándola, con valores de $\mathrm{pH}$ correspondiendo con valores altos de concentración de oxígeno disuelto y viceversa.

\section{CONCLUSIONES}

1. El Estero de Punta Banda es un antiestuario, con salinidad y temperaturas más elevadas hacia el interior que en la boca, durante todo el año.

2. La salinidad máxima registrada en julio fue de $37.36 \%$ y la temperatura máxima registrada también en julio fue de $26.0^{\circ} \mathrm{C}$.

3. Los rangos de variación de salinidad, temperatura, concentración de oxígeno disuelto y $\mathrm{pH}$ no son limitantes para el crecimiento de Crassostrea gigas, Ostrea lurida, y Ostrea edulis, pero la salinidad no permite el cultivo de Crassostrea virginica. La temperatura del extremo in- terno en verano es adecuada para el desove natural de Crassostrea gigas $Y$ Ostrea edulis.

\section{AGRADECIMIENTOS}

El presente trabajo constituye, en una versión más amplia, la tesis profesional que presentó Raúl Celis Ceseña a la Escuela Superior de Ciencias Marinas de la UABC, para obterier el título de Oceanólogo. Los autores desean agradecer la colaboración de los compañeros Oceanólogos Héctor D. Rivera Carro, Sergio R. Mora Alba, Rubén Lara Lara, Catalina López Alvarez, Manuel J. Acosta Ruiz, José Luis Sánchez Hernández y Luis A. Galindo Bect, por su colaboración en los muestreos de campo y análisis de laboratorio.

\section{BIBLIOGRAFIA}

Acosta Ruiz, M. J. y S. Alvarez Borrego. 1974, Distribución superficial de algunos parámetros hidrológicos físicos y químicos, en el Estero de Punta Banda, B. C., en otoño e invierno. Ciencias Marinas, (1) 1: 16-45.

Alvarez Borrego, S. y A. Chee Barragán. Primer reporte de los estudios bioecológicos y trabajos de ostricutura en Bahía San Quintín, B. C. II etapa, sección 1: Hidrología. Unidad de Ciencias Marinas, UABC-Dirección de Acuacultura, S.R.H. Ensenada, B. C. (no publicado).

Bardach, J. E., J. H. Ryther y W. Melarney, 1972, Aquaculture. Wiley-interscience: $\mathbf{6 7 4 - 7 4 0 .}$

Cabrera Muro, H. R. 1974. Distribución de temperatura en la Bahla de Todos los Santos (junio-octubre, 1971). Ciencias Marinas, (1) 1: 65-77.

Contreras Rivas, 1. 1973. Influencia termohalina de las aguas del Estero de Punta Banda en la Bahía de Todos Santos, B. C. Tesis profesional. Escuela Superior de Ciencias Marinas, Universidad Autónoma de Baja California. Ensenada, B. C.

Jaime Silva, D. R. 1974. Cultivo de ostión japonés (Crassostrea gigas) en el Estero de Punta Banda de la Bahla de Todos Santos. Tesis profesional. Escuela Superior de Ciencias Morinas, Universidad Autónoma de Baja Calif. Ensenada, B. C.

Lara Lara, J. R. y S. Alvarez Borrego. 1975. Ciclo anual de clorofilas y producción orgúnica primaria en Bahía San Quintín, B. C. Ciencias Marinas, este número. Loosanoff, V. L. 1965. The American or Eastern Oyster. Bull. Com. Fish., Fish and Wildlife Service, U. S. Dep. Int. Circ. 205: 35. 